\title{
Ineffective Antibiotic Prophylaxis: An Unusual Presentation of Infective Endocarditis with Insights into the Appropriateness of Prophylaxis
}

\author{
Hiren Patel ${ }^{1}$, Sundeep Kumar ${ }^{1}$, Nway L. Ko Ko ${ }^{1}$, Jelena Catania ${ }^{2}$, Aamir Javaid ${ }^{3}$ \\ 1. Internal Medicine, University of Central Florida College of Medicine, Orlando, USA 2. Internal \\ Medicine - Infectious Diseases, University of Central Florida College of Medicine, Orlando, USA 3. \\ Cardiology, University of Central Florida College of Medicine/Hospital Corporation of America Graduate \\ Medical Education Consortium, Orlando, USA
}

Corresponding author: Hiren Patel, hiren.patel@ucf.edu

\section{Abstract}

The practice of antibiotic prophylaxis for the prevention of infective endocarditis (IE) has undergone a major paradigm shift over the past few decades. From the earlier practice of antibiotic prophylaxis for all patients undergoing dental procedures, since 2006, the American College of Cardiology/American Heart Association (ACC/AHA) guidelines have now narrowed down the indications to use it only in patients at high risk for IE. A true concern for antibiotic resistance remains eminent when used without appropriate indications. Through this case presentation of IE and the associated preceding use of antibiotics, we take this opportunity to explain the appropriateness of prophylaxis in patients from different risk groups. We also shed some light on alternative, effective, and, yet, harmless measures for the prevention of IE in patients at low risk.

Received 04/21/2019

Review began 05/19/2019 Review ended 05/21/2019 Published 06/07/2019

(c) Copyright 2019

Patel et al. This is an open access article distributed under the terms of the Creative Commons Attribution License CC-BY 3.0., which permits unrestricted use, distribution, and reproduction in any medium, provided the original author and source are credited.
Categories: Cardiology, Internal Medicine, Infectious Disease

Keywords: aortic valve, endocarditis, antibiotic prophylaxis, streptococcus intermedius, streptococcus spp

\section{Introduction}

Antibiotics for the prevention of infective endocarditis (IE) have been in routine clinical practice without much supportive clinical data until the publication of the 2006 American College of Cardiology/American Heart Association (ACC/AHA) guidelines limiting indications for their role [1]. Updated guidelines over 10 years later still stress that antibiotic prophylaxis for IE should only be used in high-risk individuals [2]. Prophylactic antibiotics can fail even when the infecting organism is susceptible to antibiotics and the number needed to treat for the prevention of the very few cases of IE is relatively high [3]. In spite of the guidelines, epidemiological studies reveal the inappropriate use of antibiotics for infective endocarditis prophylaxis, including but not limited to incorrect dosing, timing, and choice of antibiotics [45].

Transient bacteremia can commonly follow dental procedures but do not always necessarily require gingival manipulation. It can also potentially occur at a low grade/intensity with inadequate dental hygiene. Reduction in bacteremia with antibiotic prophylaxis has not prevented IE significantly [6]. In fact, inappropriate antibiotic use in such cases can lead to antibiotic resistance [2].

\section{How to cite this article}

Patel H, Kumar S, Ko Ko N L, et al. (June 07, 2019) Ineffective Antibiotic Prophylaxis: An Unusual Presentation of Infective Endocarditis with Insights into the Appropriateness of Prophylaxis. Cureus 11(6): e4860. DOI 10.7759/cureus.4860 
According to the ACC/AHA Task Force as of 2017, there have been no randomized controlled trials performed to show the benefit of antibiotic prophylaxis in low-risk patients [2]. A key concern is educating patients about the appropriate indications, dosing, and timing of effective antibiotic prophylaxis prior to the procedure. Adequate dental hygiene is important to prevent continuous low-grade bacteremia (potential etiology for IE) with effective, high-potency mouthwashes and optimum oral hygiene to control viridans group streptococci.

\section{Case Presentation}

A 53-year-old African American female with well-controlled non-insulin dependent diabetes mellitus (hemoglobin A1c 5.8) presented to the emergency department with a fourweek history of generalized fatigue, malaise, and $20 \mathrm{lb}$ weight loss, along with a two-week history of right upper quadrant pain, subjective fever, nausea, and vomiting. The patient underwent dental root extraction five weeks prior to admission. Although she did not have a prior history of endocarditis or a prosthetic valve, she was given one dose of two grams amoxicillin for endocarditis prophylaxis, which she took the day before the procedure. On arrival, the patient was in sepsis, with a temperature of $101.9^{\circ} \mathrm{F}$ and blood pressure of $96 / 60$ $\mathrm{mm} \mathrm{Hg}$ but attained hemodynamic stability after appropriate fluid resuscitation. Physical examination was significant for tender hepatomegaly.

Initial lab results showed leukocytosis with left shift, normocytic anemia, elevated transaminases, hyperbilirubinemia, coagulopathy, and acute kidney injury (See Table 1 for lab results). She was immediately started on empiric broad-spectrum intravenous (IV) antibiotics after the initial blood cultures were drawn. Initial imaging included a liver ultrasound that revealed multiple hepatic lesions, confirmed with abdominal computed tomography (CT). Magnetic resonance cholangiopancreatography (MRCP) confirmed the absence of common bile duct dilation (See Figure $1 A-1 B$ for imaging findings). She had negative workup for both viral hepatides and tumor markers for gastrointestinal tumors. A CT-guided needle biopsy of hepatic lesions revealed purulent fluid with a white blood cell (WBC) count $>170,000 / \mathrm{mm}^{3}$, negative for ova and parasites. The initial blood cultures drawn on admission and the fluid aspirate from hepatic lesions both grew Streptococcus intermedius (gram-positive cocci in clusters) with low penicillin minimal inhibitory concentration (MIC). The patient subsequently underwent a transesophageal echocardiogram revealing a one-centimeter aortic valvular vegetation without associated valvular dysfunction (Figure $1 C-1 E$ ). 


\section{Cureus}

$\begin{array}{llll}\text { Laboratory findings } & & & \\ \text { WBC } & 15.82 \mathrm{~K} / \mathrm{mm}^{3} & \text { Albumin } & 2.3 \mathrm{gm} / \mathrm{dL} \\ \text { Creatinine } & 2.17 \mathrm{mg} / \mathrm{dL} & \text { Total Protein } & 6.5 \mathrm{gm} / \mathrm{dL} \\ \text { eGFR } & 29 \mathrm{ml} / \mathrm{min} & \text { PT } & 16.5 \mathrm{sec} \\ \text { AST } & 387 \mathrm{unit} / \mathrm{L} & \text { INR } & 1.57 \\ \text { ALT } & 235 \mathrm{unit} / \mathrm{L} & \text { ESR } & 104 \mathrm{~mm} / \mathrm{hr} \\ \text { Total Bilirubin } & 3.3 \mathrm{mg} / \mathrm{dL} & \text { CRP } & 28 \mathrm{mg} / \mathrm{dL} \\ \text { Direct Bilirubin } & 2.2 \mathrm{mg} / \mathrm{dL} & \text { Procalcitonin } & 21.59 \mathrm{ng} / \mathrm{mL} \\ \text { Alkaline Phosphatase } & 108 \mathrm{unit} / \mathrm{L} & \end{array}$

\section{TABLE 1: Laboratory findings on initial presentation}

ALT, Alanine Transaminase; AST, Aspartate Transaminase; CRP, C - Reactive Protein; eGFR, effective Glomerular Filtration Rate; ESR, Erythropoietin Sedimentation Rate; INR, International Normalized Ratio; PT, Prothrombin Time; WBC, White Blood Cells

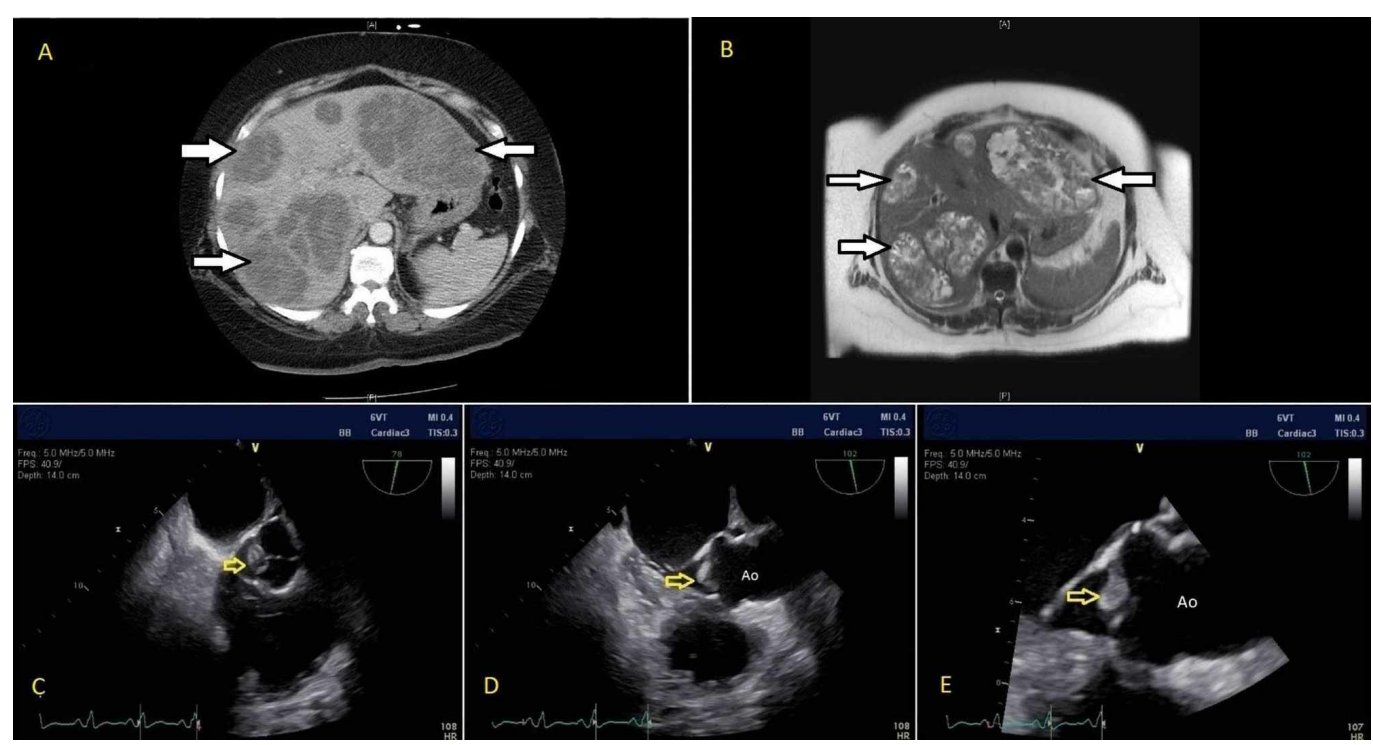

FIGURE 1: (A) CT abdomen and (B) MRCP finding of multiple hepatic abscesses (solid white arrows); (C-E) Transesophageal echocardiographic findings of aortic valvular vegetation (yellow arrows)

Ao, Aorta; CT, Computed Tomography; MRCP, Magnetic Resonance Cholangiopancreatography

The patient clinically improved and cleared blood cultures within 24 hours of abscess drainage and appropriate IV antibiotics. There was interval improvement in her renal and liver function 


\section{Cureus}

tests, as noted in Table 2. She was discharged on intravenous antibiotics to complete a sixweek course. Upon discharge, she was strongly recommended to take infective endocarditis prophylaxis during any future dental procedures involving mucosal manipulation and to take her antibiotic one hour prior to the procedure.

$\begin{array}{lcc}\begin{array}{l}\text { Interval improvement in pertinent laboratory findings } \\ \text { Laboratory findings }\end{array} & \text { On admission } & \text { On discharge } \\ \text { AST } & 387 \mathrm{unit} / \mathrm{L} & 53 \mathrm{unit} / \mathrm{L} \\ \text { ALT } & 235 \mathrm{unit} / \mathrm{L} & 28 \mathrm{unit} / \mathrm{L} \\ \text { Total Bilirubin } & 3.3 \mathrm{mg} / \mathrm{dL} & 1.5 \mathrm{mg} / \mathrm{dL} \\ \text { Direct Bilirubin } & 2.2 \mathrm{mg} / \mathrm{dL} & 1.2 \mathrm{mg} / \mathrm{dL} \\ \text { Creatinine } & 2.17 \mathrm{mg} / \mathrm{dL} & 0.84 \mathrm{mg} / \mathrm{dL}\end{array}$

TABLE 2: Laboratory findings (improved) at the time of hospital discharge

ALT, Alanine Transaminase; AST, Aspartate Transaminase

\section{Discussion}

Streptococcus intermedius has been isolated from patients with periodontitis and can cause fatal purulent infections, especially brain and liver abscesses following dental procedures [7-9], but this is an unusual presentation culminating in endocarditis with an accompanying liver abscess. Usually, the most common Streptococcus (S) viridans group organisms known to cause IE are S. sanguinis, S. bovis, S. mutans, and S. mitis and the S. salivarius group that cumulatively accounts for the majority of native valve endocarditis [10-11] while S. intermedius (from the Streptococcus anginosus group) has a relatively lower incidence.

Although there have been limited cases of Streptococcus endocarditis documented after dental procedures, the most common clinical presentation after such procedures is that of bacteremia [12]. Transient bacteremia can occur after routine teeth brushing and does not necessarily require gingival manipulation. Reduction in bacteremia with antibiotic prophylaxis has not prevented infective endocarditis significantly [6]. Over the past decade (2006 - 2017), we have seen constant efforts by the American College of Cardiology (ACC) and American Heart Association (AHA) to address this clinical decision regarding IE prophylaxis. It has been shown that inappropriate antibiotic use in many cases can increase the risk of antibiotic resistance and there have been no randomized controlled trials performed to show the benefit of antibiotic prophylaxis in patients with a low risk of IE [2]. A nationwide population-based cohort and a case crossover study by Tubiana and colleagues suggest that invasive dental procedures may contribute to a very small number of cases of bacterial endocarditis, but the benefit of antibiotic prophylaxis remains uncertain and likely minuscule [13].

In this case, our patient did not meet the criteria for IE prophylaxis for the dental procedure. Her dental exam during this hospitalization revealed generalized mild periodontitis with no active abscess at the site of dental root extraction. However, despite receiving the IE prophylaxis prior to the dental procedure (amoxicillin two grams once), she developed 
endocarditis as the effective duration of action of administered antibiotic had already elapsed (half-life of 60 minutes with time to peak ranging from one to two hours and is excreted via urine within six to eight hours). Guidelines provide recommendations regarding the patient population at risk, the choice and the timing of such antibiotics (one hour before the procedure) [14]. Hence, it is a cornerstone step in explaining to patients the importance of prophylaxis timing (if indicated).

We take this opportunity to pose another critical question: how do we reduce the possibility of IE in low-risk patients without the use of antibiotics? A 2014 study by Elshibly et al. showed that simple oral hygiene with a total of one-minute exposure to high-potency mouthwashes killed viridans group streptococci organisms and suggested a significant reduction of IE [15]. A study by Veloso et al. used rats as the animal model for the induction of experimental endocarditis with continuous low-grade and intermittent high-grade bacteremia. It showed that both these mechanisms potentially induced experimental endocarditis. This data support the hypothesis that cumulative exposure to low-grade bacteremia (from longstanding inadequate dental hygiene) does represent a genuine risk of IE in humans [16]. However, these are single-center in-vitro studies lacking external validation and applicability to the large-scale human population. Regardless of individual risk factors, it is crucial to emphasize regular and thorough dental hygiene to all patients undergoing dental procedures. This is a low-level intervention without harm but may be beneficial, unlike antibiotic use for prophylaxis. Prophylaxis can only be an additive effect with daily dental hygiene for individuals with a high risk for IE.

In the end, we pose several important questions through this case review:

1. What is the effective role of IE prophylaxis after all?

2. Mechanism of bacteremia, continuous low-grade (associated with inadequate regular dental hygiene) versus high-grade bacteremia (associated with dental procedures like gingival manipulation) or both?

\section{Conclusions}

Transient bacteremia can commonly follow dental procedures but does not always necessarily require gingival manipulation. Reduction in bacteremia with antibiotic prophylaxis has not prevented IE significantly, but inappropriate antibiotic use (especially in low-risk patients) can potentially lead to antibiotic resistance. There have been no randomized controlled trials performed to show the benefit of antibiotic prophylaxis in low-risk patients, thus needing further studies in this perspective. A key concern is correctly identifying the population at risk and educating them about appropriate indications and the timing of effective antibiotic prophylaxis prior to the procedure. Until then, adequate dental hygiene on a regular basis is important to prevent continuous low-grade bacteremia (potential etiology for IE) to control viridans group streptococci.

\section{Additional Information}

\section{Disclosures}

Human subjects: Consent was obtained by all participants in this study. Conflicts of interest: In compliance with the ICMJE uniform disclosure form, all authors declare the following:

Payment/services info: All authors have declared that no financial support was received from any organization for the submitted work. Financial relationships: All authors have declared that they have no financial relationships at present or within the previous three years with any organizations that might have an interest in the submitted work. Other relationships: All 
authors have declared that there are no other relationships or activities that could appear to have influenced the submitted work.

\section{Acknowledgements}

We would like to thank Dr. Rick Nishimura (Chair, Division of Structural Heart Disease, Department of Cardiovascular Medicine, Mayo Clinic, MN) for critical appraisal of the final manuscript. Dr. Rick Nishimura is an expert and has contributed to the major clinical guidelines for valvular heart disease over two decades.

\section{References}

1. Bonow RO, Carabello BA, Chatterjee K, et al.: ACC/AHA 2006 guidelines for the management of patients with valvular heart disease: a report of the American College of Cardiology/American Heart Association Task Force on practice guidelines (Writing Committee to revise the 1998 guidelines for the management of patients with valvular heart disease) developed in collaboration with the Society of Cardiovascular Anesthesiologists endorsed by the Society for Cardiovascular Angiography and Interventions and the Society of Thoracic Surgeons. J Am Coll Cardiol. 2006, 48:1-148. 10.1016/j.jacc.2006.05.021

2. Nishimura RA, Otto CM, Bonow RO, et al.: 2017 AHA/ACC focused update of the 2014 AHA/ACC guideline for the management of patients with valvular heart disease: a report of the American College of Cardiology/American Heart Association Task Force on Clinical Practice Guidelines. J Am Coll Cardiol. 2017, 70:252-289. 10.1016/j.jacc.2017.03.011

3. Duval X, Alla F, Hoen B, et al.: Estimated risk of endocarditis in adults with predisposing cardiac conditions undergoing dental procedures with or without antibiotic prophylaxis. Clin Infect Dis. 2006, 42:102-107. 10.1086/504385

4. Duval X, Delahaye F, Alla F, et al.: Temporal trends in infective endocarditis in the context of prophylaxis guideline modifications: three successive population-based surveys. J Am Coll Cardiol. 2012, 59:1968-1976. 10.1016/j.jacc.2012.02.029

5. DeSimone DC, Tleyjeh IM, Correa de Sa DD, et al.: Incidence of infective endocarditis due to viridans group streptococci before and after the 2007 American Heart Association's Prevention Guidelines: an extended evaluation of the Olmsted County, Minnesota, population and Nationwide Inpatient Sample. Mayo Clin Proc. 2015, 90:874-881.

10.1016/j.mayocp.2015.04.019

6. Roberts GJ, Radford P, Holt R: Prophylaxis of dental bacteraemia with oral amoxycillin in children. Br Dent J. 1987, 162:179-182. 10.1038/sj.bdj.4806069

7. Corredoira J, Casariego E, Moreno C, et al.: Prospective study of Streptococcus milleri hepatic abscess. Eur J Clin Microbiol Infect Dis. 1998, 17:556-560. 10.1007/BF01708618

8. Molina JM, Leport C, Bure A, Wolff M, Michon C, Vilde JL: Clinical and bacterial features of infections caused by Streptococcus milleri. Scand J Infect Dis. 1991, 23:659-666. $10.3109 / 00365549109024289$

9. Bert F, Bariou-Lancelin M, Lambert-Zechovsky N: Clinical significance of bacteremia involving the "Streptococcus milleri" group: 51 cases and review. Clin Infect Dis. 1998, 27:385387. 10.1086/514658

10. Murdoch DR, Corey GR, Hoen B, et al.: Clinical presentation, etiology, and outcome of infective endocarditis in the 21st century: the International Collaboration on EndocarditisProspective Cohort Study. Arch Intern Med. 2009, 169:463-473. 10.1001/archinternmed.2008.603

11. Sunnerhagen T, Tornell A, Vikbrant M, Nilson B, Rasmussen M: HANDOC: a handy score to determine the need for echocardiography in non-beta-hemolytic streptococcal bacteremia. Clin Infect Dis. 2018, 66:693-698. 10.1093/cid/cix880

12. Cahill TJ, Harrison JL, Jewell P, et al.: Antibiotic prophylaxis for infective endocarditis: a systematic review and meta-analysis. Heart. 2017, 103:937-944. 10.1136/heartjnl-2015309102

13. Tubiana S, Blotière P-O, Hoen B, et al.: Dental procedures, antibiotic prophylaxis, and endocarditis among people with prosthetic heart valves: nationwide population based cohort and a case crossover study. BMJ. 2017, 358:10.1136/bmj.j3776 


\section{Cureus}

14. Wilson W, Taubert KA, Gewitz M, et al.: Prevention of infective endocarditis: guidelines from the American Heart Association: a guideline from the American Heart Association Rheumatic Fever, Endocarditis, and Kawasaki Disease Committee, Council on Cardiovascular Disease in the Young, and the Council on Clinical Cardiology, Council on Cardiovascular Surgery and Anesthesia, and the Quality of Care and Outcomes Research Interdisciplinary Working Group. Circulation. 2007, 116:1736-1754. 10.1161/circulationaha.106.183095

15. Elshibly A, Coulter WA, Millar BC, et al.: Effective oral health in infective endocarditis: efficacy of high-street mouthwashes against the viridans group streptococci. J Investig Clin Dent. 2014, 5:151-153. 10.1111/jicd.12088

16. Veloso TR, Amiguet M, Rousson V, Giddey M, Vouillamoz J, Moreillon P, Entenza JM: Induction of experimental endocarditis by continuous low-grade bacteremia mimicking spontaneous bacteremia in humans. Infect Immun. 2011, 79:2006-2011. 10.1128/iai.01208-10 